\title{
Energy Aware Battery Powered Electric Vehicles:A Predictive Model Driven Approach
}

\author{
${ }^{1}$ Dennis Babu, ${ }^{2}$ Anirudh Kumar and ${ }^{3}$ Joydeb Roychowdhury \\ Embedded Systems Laboratory \\ CSIR- Central Mechanical Engineering Research Institute \\ Durgapur, West Bengal, India \\ 1'dennis.babu@cmeri.res.in, ${ }^{2}$ anirudhkumar@cmeri.res.in and ${ }^{3}$ jrc@cmeri.res.in
}

\begin{abstract}
Active energy management for power optimization is essential for successful commercialization of battery powered electric vehicles. In this paper we propose and implement an energy aware electric vehicle using an efficient battery management system, EffBMS which estimates the remaining charge of the $\mathrm{Li}$-ion battery online, learns the rate of change of SOC and accordingly modulates the velocity and acceleration of the vehicle for optimal energy usage. An Extended Kalman Filter (EKF) was used to estimate the remaining charge of the Li-ion battery pack. An online dual time frame gradient descent algorithm with higher time frame for updating dataset and lower time frame for parameter tuning learns the SOC curve and employs a set point controller for energy management in motion. The entire model was developed in a SPARTAN $6 \mathrm{LX}-45$ reconfigurable FPGA board and is tested in a laboratory model of differentially driven single geared electric car. Case study of an upward slope hill motion is performed and results shows $10-12 \%$ energy savings.(Abstract)
\end{abstract}

Keywords-; battery management system; FPGA; gradient descent algorithm; Extended Kalman Filter; EffBMS

\section{INTRODUCTION}

The rising fuel price and green house emissions have prompted the automobile industry to shift the focus on electric vehicles (EVs) and hybrid electric vehicles (HEVs). The national vehicle plan launched in 2013 aims to sell 6-7 million EVs in India by 2020[1]. Batteries and battery management system (BMS) are an important part of the EVs as it determines the critical specifications of the vehicle such as maximum range, max acceleration and velocity. Dynamic load scheduling for power optimization and range extension is essential for successful commercialization of EVs. Such dynamic load scheduling requires perfect knowledge of the road profile and traffic conditions with minimal sensor data. Battery data driven power optimization is more effective as road profile, traffic conditions and driver instincts are reflected in battery data..

SOC estimation is the most basic requirement of an active energy management system. Coulomb counting [2] and dynamic battery models [3] were proposed to estimate the SOC of the EV battery. Artificial neural networks [4] and Support Vector machines [5] were also employed for remaining charge estimation. Battery Modeling errors coupled with initialization of coulomb counting led to estimation tools such as Extended Kalman filter and Unscented Kalman filter [6].

Several energy management strategies have been proposed in the literature till date. Driving pattern detection based multimode vehicle control is discussed in [7].Road terrain preview based energy management is proposed in [8], where as multi feature statistical approach for distinguishing driving patterns are discussed in [9]. Darnelli proposed a modified SOC tracking considering the driving conditions based on cartographic data [10]

In this paper we propose an energy aware battery management system which estimates the SOC of the battery online using an online EKF algorithm. A machine learning framework using online batch gradient descent algorithm further learns the discharge profile of battery from SOC. This model predictive strategy is further used to modulate the driver control of the vehicle using a set point controller for optimizing the energy consumption. The battery pack used for the study is a Li-ion $7.4 \mathrm{~V}$ 4.4Ah battery pack. The EffBMS is implemented in a SPARTAN 6 LX-45 FPGA board making it readily reconfigurable. The hardware architecture of EffBMS and constituent blocks are discussed. A scaled down laboratory model of electric car is used to validate the proposed methodology. The upward slope road profile is the critical energy consuming phase of electric vehicle drive cycle, thus the proposed methodology is analyzed and validated in the above profile.

The rest of the paper is organized as follows. Section II presents the SOC estimation framework. Section III illustrates the learning mechanism and active energy management strategy. The Hardware architecture of EffBMS is detailed in section IV, while real world results are discussed in section $\mathrm{V}$, followed by conclusion in section VI.

\section{REMAINING CHARGE ESTIMATION}

The SOC is estimated using a novel combination of EKF algorithm, Thevenins battery model and coulomb counting method. The frame work of estimation is given as follows.

\section{A. Battery model}

The battery model should incorporate the actual Li-ion battery characteristics such as internal resistance, double layer and charge transfer and diffusion. The double layer and charge transfer are high frequency characteristics with time period less than $0.02 \mathrm{sec}$ while diffusion is modeled as an infinite sequence of resistance-capacitance pairs of different 
time periods [11]. The exact battery model is thus practically impossible to implement in a real time embedded systems. Thus we go for a simplified battery model, Thevenins first order battery model [3] consisting of an internal resistance, Rin and a series resistance capacitance pair, Rc-Cc which models the slow dynamics of the battery. The fast dynamics such as double layer-charge transfer and high frequency components of diffusion are taken care in the EKF noise model. Fig. 1 shows the battery model and subsequently state equation and measurement function is derived in (1)-(6).

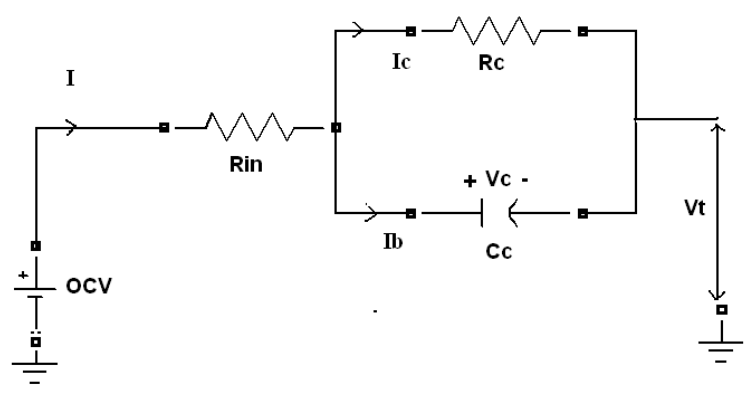

Figure 1: Battery model

From Kirchhoff's voltage law, measurement function

$$
V t=O C V-I R_{i n}-V_{c}
$$

Again applying Kirchhoff's current law,

$$
I=I_{b}+I_{c}
$$

which implies state equation as

$$
I=\frac{V_{c}}{R_{c}}+C \frac{d V_{c}}{d t}
$$

Solving for the value of $\mathrm{Vc}$,

$$
V_{c}=A e^{\frac{-t}{R_{c} C_{c}}}+I R_{c}
$$

Where $A$ is the constant value which could be found from initial conditions. Under zero initial conditions the equation (4) reduces to

$$
A=-I R_{c}
$$

Thus combining zero state voltage and zero input voltage the net voltage is given by

$$
V_{c}=V_{c}(0) e^{\frac{-t}{R_{c} C_{c}}}+R_{c}\left(1-e^{\frac{-t}{R_{c} C_{c}}}\right)
$$

The initial battery parameters are found out by using standard Hybrid pulse profile cycle test [12] where battery was charged at $.75 \mathrm{C}$ for 10 seconds followed by rest period of 40 seconds and further discharge for 10 seconds at $1 \mathrm{C}$. The actual battery profile obtained in the above experiment is used to fit the exponential curve [13] using the Lavenberg Marquedet least squares fitting. The initial battery parameters of the model where found to be Rin $=0.025 \mathrm{ohms}$, $\mathrm{Rc}=0.02 \mathrm{ohms}$ and $\mathrm{Cc}=550 \mathrm{pF}$.

\section{B. Coulomb counting and SOC-OCV table}

The coulomb counting model to be implemented in the discretized form is given by (7) where $\eta$ is the coulombic efficiency found out by the ratio of energy consumed by the battery to that delivered across any load. The $\eta$ value fall in the range of $[0-1]$ and was found to be $\approx 1$ for the $\mathrm{Li}$-ion battery under test.

$$
\operatorname{SOC}(k)=\operatorname{SOC}(k-1)-\frac{\eta \Delta t I_{b}}{C_{h}}
$$

\section{EKF framework}

An accurate noise model which compensates the modeling errors and measurement errors improves the SOC estimation. Kalman filtering provides an elegant and powerful solution for state estimation which has been widely used in the past in various fields such as target tracking, dynamic system control etc. The generic definition of any dynamic system with input $\mathrm{u}(\mathrm{k})$,output $\mathrm{y}(\mathrm{k})$ and states $\mathrm{x}(\mathrm{k})$ is given in (8).

$$
\begin{aligned}
& x(k)=f(x(k-1), u(k-1))+w(k-1) \\
& y(k)=g(x(k), u(k))+v(k)
\end{aligned}
$$

Here $\mathrm{v}$ and $\mathrm{w}$ are the process noise and measurement noise which are assumed to be zero mean Gaussian stochastic processes, with covariance matrixes R and Q. For efficient online implementation, a discretized mathematical description of the continuous time battery dynamics described earlier is necessary which is given in (9)-(10). The system definition of Kalman filter with state vector $\mathrm{x}$ given by $x=[S O C, V C]^{T}$ is as,

$$
\begin{gathered}
f(x)=\left[\begin{array}{cc}
1 & 0 \\
0 & \exp ^{\frac{-\Delta t}{R_{c} C_{c}}}
\end{array}\right]\left[\begin{array}{c}
\operatorname{SOC}(k-1) \\
V_{c}(k-1)
\end{array}\right]+\left[\begin{array}{c}
\frac{-\eta \Delta t}{C_{h}} \\
\frac{-\Delta t}{R_{c}\left(1-\exp ^{\frac{-}{R_{c} C_{c}}}\right)}
\end{array}\right] I(k-1) \\
g(x)=O C V-V C-I R_{i n}-V_{t}
\end{gathered}
$$

The OCV for the measurement transition function is obtained from the one to one SOC-OCV mapping obtained for the battery under study. Polynomial curve fitting is used to obtain a mathematical function of OCV in terms of SOC as given in (11).

$O C V=1.503(S O C)^{3}-2.347(S O C)^{2}+2.051 S O C+6.732(6)$

Since the system under consideration is nonlinear, we use an extended Kalman filtering for SOC estimation in which the system is linearized at all evaluation points using first order Taylor series given by, $\mathrm{A}(\mathrm{k})$ as the linearized state transition matrix and $\mathrm{C}(\mathrm{k})$ as the linearized measurement transition matrix. Accurate definition of $\mathrm{R}$ and $\mathrm{Q}$ is necessary as incorrect values may make the estimation divergent. The correlation of the error sequences between model output and actual output for a fixed duration is used to tune the values of $\mathrm{R}$ and Q [14]. The "predict" and "correct" formulation of the 2 state EKF is further implemented [11].

\section{PREDICTIVE ENERGY MANAGEMENT BY LEARNING}

\section{A. Gradient descent based SOC learning}

The unsupervised learning is implemented online to track the rate of change of SOC. The estimated SOC from the EKF was stored in a queue of length N. Data in queue was learned by Batch gradient descent algorithm to predict the future values of SOC. 
As the process is recursive the time required to fit the data points increases with the number of data points. Thus it is essential to ensure the global minima fitting of the present data set before the updation with next data points. To ensure the above goal we device a dual time frame learning mechanism in which we update the dataset in a higher time frame, Th (here 5 seconds) and recursively iterate the tuning process of the data in a much lower time frame, $\mathrm{Tl}$ (here 20 $\mathrm{msec}$ ). Thus this dual time frame tuning ensures that the minimum amount of iteration, necessary for global minima fitting is attained within Th.

The generic cost function for learning is the least means square algorithm as it ensures the global minima [15] avoiding any local minima in the tuning process. The hypothesis of gradient descent tuning is described as,

$$
h_{x}(t)=\sum_{i=0}^{n} x_{i} t_{i}=x^{T} t
$$

The generic cost function is given by

$$
J(x)=\frac{1}{2} \sum_{i=1}^{N}\left(h_{x}\left(t_{i}\right)-y_{i}\right)^{2}
$$

Where $h_{x}\left(t_{i}\right)$, is the hypothesis as a function of time $t$, $y_{i}$ is the data set to be fitted with time and $\mathrm{N}$ is the length of the data set. A straight line fitting is used to track SOC which gives intercept and slope of SOC degradation at each point of time. Thus the modified hypothesis and cost function specific to EffBMS is given by (14)-(15)

$$
\begin{gathered}
h_{x}(t)=x_{0}+x_{1} t \\
J(x)=\frac{1}{2} \sum_{i=1}^{N}\left(x_{0}+x_{1} t_{i}-S O C_{i}\right)^{2}
\end{gathered}
$$

Where $x_{o}$ is the intercept, $x_{1}$ is the gradient of the slope and $S O C_{i}$ is the $\mathrm{SOC}$ at the ith time instant. The generic gradient descent parameter updation given in (24) reduces to the specific case as ,

$$
x_{j}=x_{j}-\alpha \frac{\partial}{\partial x_{j}}(J(x))
$$

Where

$$
\frac{\partial}{\partial x_{j}}(J(x))=\left(h_{x}(t)-S O C\right) t_{j}
$$

Thus the individual tuning of the parameters are done by

$$
x_{0}=x_{0}+\alpha \sum_{i=1}^{N}\left(S O C_{i}-h_{x}\left(t_{i}\right)\right)
$$

And

$$
x_{1}=x_{1}+\alpha \sum_{i=1}^{N}\left(S O C_{i}-h_{x}\left(t_{i}\right)\right) t_{i}
$$

\section{B. Prediction of Remaining time of operation of battery}

At any point the rate of change of SOC will give the approximate remaining time of operation of the battery under similar load conditions. Thus the learned SOC from the batch gradient descent is extrapolated to predict the SOC after time, t. From (14)

$$
\operatorname{SOC}\left(t_{h}\right)=x_{0}+x_{1} t_{h}
$$

$\operatorname{SOC}\left(t_{h}\right)$, is the SOC after time $t_{h}$ from the learned parameters, $x_{0}$ and $x_{1}$ from the learning module. Thus the remaining time of operation of battery, $t_{r}$ is given by

$$
t_{r}=\frac{-x_{0}}{x_{1}}
$$

\section{Set point control based predictive energy management system}

The predicted SOC is further used to modulate the velocity and acceleration of the vehicle to optimize the total energy usage. SOC is the limiting factor which restricts the peak power ratings of the battery and thus the peak acceleration and velocity of the vehicle. The energy required for uphill motion of the vehicle is more than that of the flat profile road [10].The sudden acceleration applied by the driver in the uphill terrain will drain the battery. Thus energy management is important during the uphill motion of the vehicle.

The EffBMS has an iterative sequence of "learning" and "action" phases in a MASTER-SLAVE architecture where controller learns the SOC variation parameters ( $\mathrm{x} 0$ and $\mathrm{x} 1$ ) in the learning phase and varies the speed of the vehicle based on the learned parameters using pulse width modulation in the action phase. The entire pulse period is

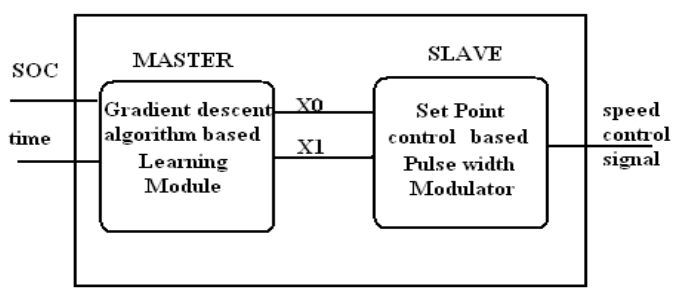

Figure 2: Set point controller schematic

divided into 256 levels and the pulse width is decreased or increased based on the different set points based on the learned parameters thereby modulating the actual driver control input. The block schematic of the control scheme is given in Fig. 2. 


\section{HARDWARE ARCHITECTURE OF EFFBMS}

The EffBMS was tested and validated in a scaled down laboratory model of an electrical car (LMEC) weighing 20 $\mathrm{Kg}$ shown in Fig. 3 with in-wheel integrated fixed gearing motors and differential drive for direction control. The LMEC has a $4.4 \mathrm{Ah} 7.4 \mathrm{~V}$ battery pack as the energy source with high power, high torque DC motor traction.

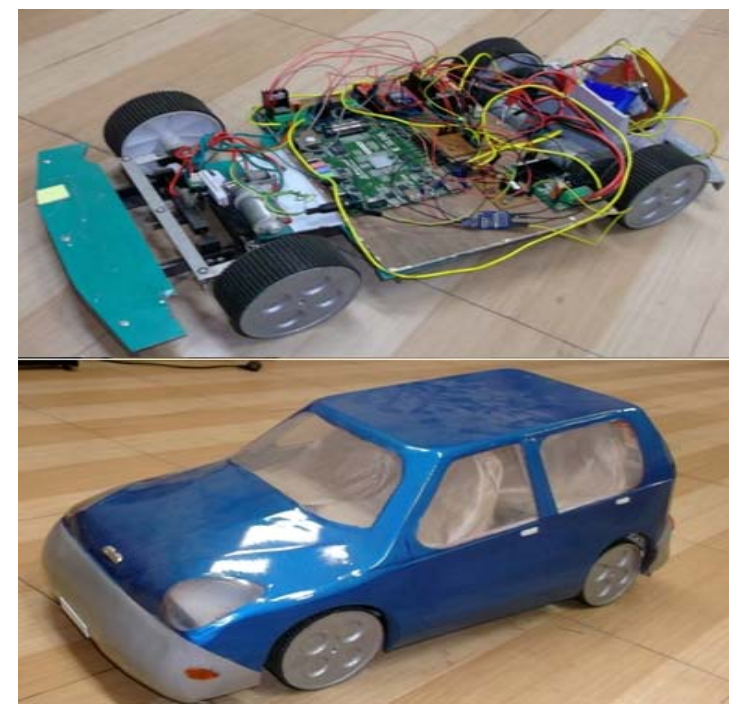

Figure 3: Laboratory model of electric car (LMEC)

$\mathrm{A} \pm 5 \mathrm{~A}$ Hall Effect current sensor with $0.1 \%$ accuracy has been used for current sensing. The battery charging set up consists of an Agilent E3633A programmable DC power supply with constant voltage and constant current modes and a $60 \mathrm{MHz}$ Tektronix TDS 1002B two channel digital storage oscilloscope. The FPGA chip of the experiment board is LX-45 (speed grade-3) which is Spartan 6 series of Xilinx chip with 6822 slices and 324 I/O pins. A floating point coprocessor UM FPU V3.1 is used for floating point computation thus making active energy management and estimation process parallel.

The high level hardware architecture schematic of the BMS is given in Fig. 4. The hardware architecture of the circuit designed on FPGA chip consists of an ADC interface module which collects battery voltage and current from ADC AD7991.The 12 bit binary output from the ADC interface module is converted to IEEE 32 bit floating point format using the binary to floating point converter module. The $100 \mathrm{MHz}$ CMOS clock signal, clk is reduced to a lower frequency, clk fs by the clock divider module which is used

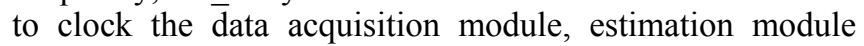
and active energy management module of EffBMS. The dual time frame gradient descent learning requires a lower frequency obtained from the clock divider 2 module. An SPI based floating point coprocessor interface module controls the data transfer between the estimation module and UM FPU V3.1 floating point coprocessor. The IEEE 801.15.4 Zigbee interface module controls the Xbee RF transceiver

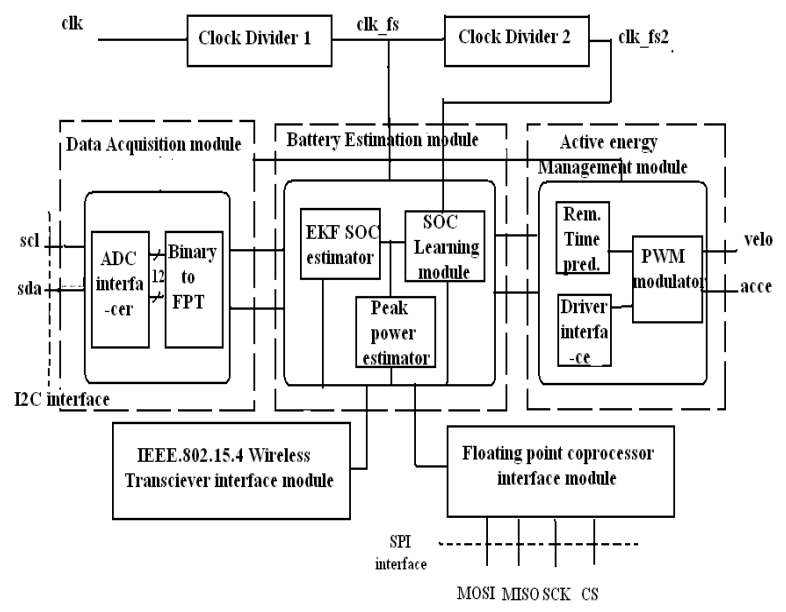

Figure 4: FPGA Hardware architecture of EffBMS

module which transmits the estimation data and learned parameters to a remote computer system for data analysis and validation. The active energy management module consisting of pulse width control module, driver interface module and remaining time estimator uses set point controller for dynamic velocity modulation.

\section{RESULTS AND DISCUSSIONS}

\section{A. SOC estimation validation}

Two test conditions were used to validate the online SOC estimation of EffBMS. Short cycle dynamic speed profile and long cycle constant speed profile test. In short cycle dynamic profile test the vehicle is driven for a short duration of 300 seconds. The estimation is validated using an accurate coulomb counter. Although coulomb counting has initialization error and cumulative accumulation error, it works fairly accurate for short duration [12]. Fig. 5 shows the comparative plot of EKF based online estimated SOC and actual SOC

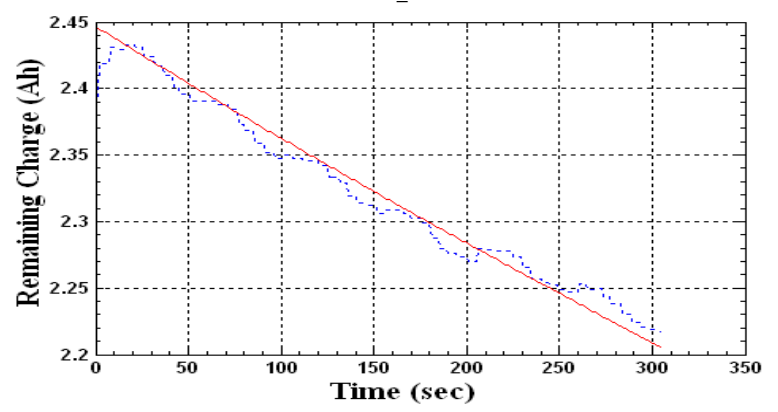

Figure 5: Coulomb counting based SOC validation

In the long cycle test the battery is operated in its entire operational regions .85-.25 SOC under variable road profile. Coulomb counting cannot be used since integral error piles up since the duration of test is long. Hence we use the OCV 
method in which the battery is open circuited for 2 hours at preset test points for validating the online estimation [11]. The open circuit voltage of the battery after 2 hours bears one to one mapping with $\mathrm{SOC}$ which is used to provide actual SOC. Fig. 6 shows the plot of online SOC with experimental SOC validation points marked along it.

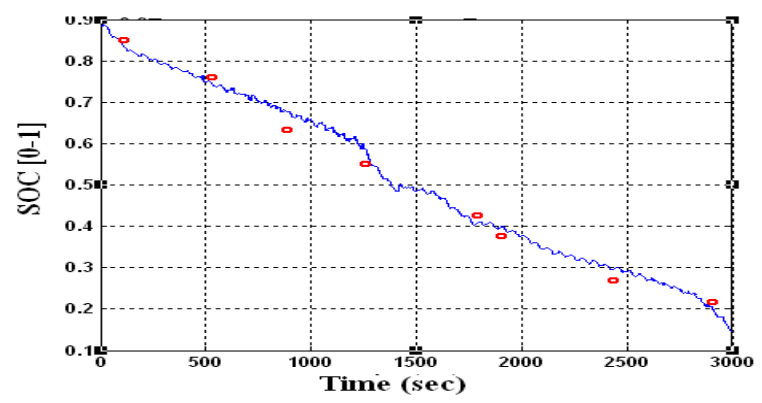

Figure 6: OCV based SOC validation

\section{B. Remaining time estimation and validation}

The gradient descent algorithm estimates the remaining time of operation of the battery based on extrapolation of learned behavior of the battery performance. Fig. 7 shows the plot of the remaining time of operation for the SOC profile given in the insight of the same. The actual run time of the LMEC is 2100 seconds but the initial prediction of 2000 seconds was updated in latter phase of learning process, updating the prediction on each iteration.

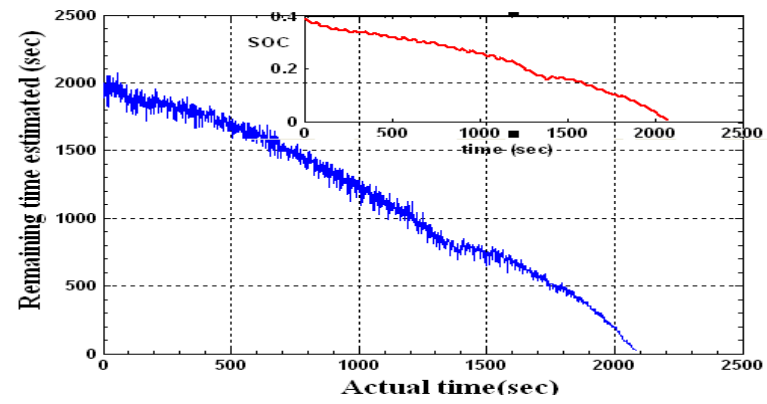

Figure 7: Remaining time estimation

\section{Active energy management system validation}

The active energy management based on learned battery SOC data is used to optimize the energy consumption across an uphill slope of 25 degree. Fig. 8 shows the learned parameter $\mathrm{x} 0$, which is the rate of change of $\mathrm{SOC}$ in different profiles. The magnitude of $\mathrm{x} 0$ is higher for a slope profile than for a level profile which is utilized to device the set point controller which increases the pulse width incrementally in steps to optimize the energy to travel a particular distance.

Figs. 9-12 shows the remaining charge content of the battery in different profiles and driving conditions. Fig. 9 shows the remaining charge in a level profile for a distance of 5 meters and Figs. 10-11 shows the SOC in 25 degree upward slope profile for the same distance at $12 \mathrm{~km} / \mathrm{hr}$ and 6 $\mathrm{km} / \mathrm{hr}$ respectively. While the charge consumed for level profile is only 0.10 Ah the charge consumed for $12 \mathrm{~km} / \mathrm{hr}$ and $6 \mathrm{~km} / \mathrm{hr}$ speed in 25 degree slope profile is 0.22 and 0.2 Ah respectively. Fig. 12 shows the SOC profile with the proposed algorithm which shows an energy usage of 0.175 Ah. The nonlinear rate of change of SOC shows the incremental change in the speed of the vehicle thereby reaching the required distance with minimal energy.

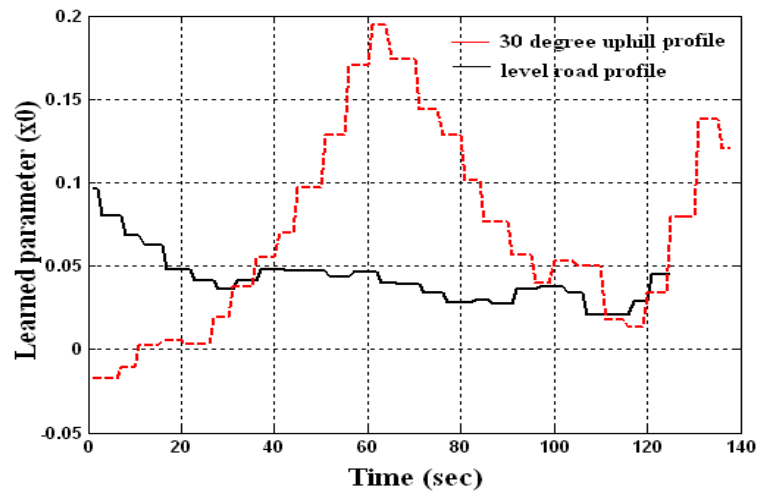

Figure 8: Parameter variation in runtime

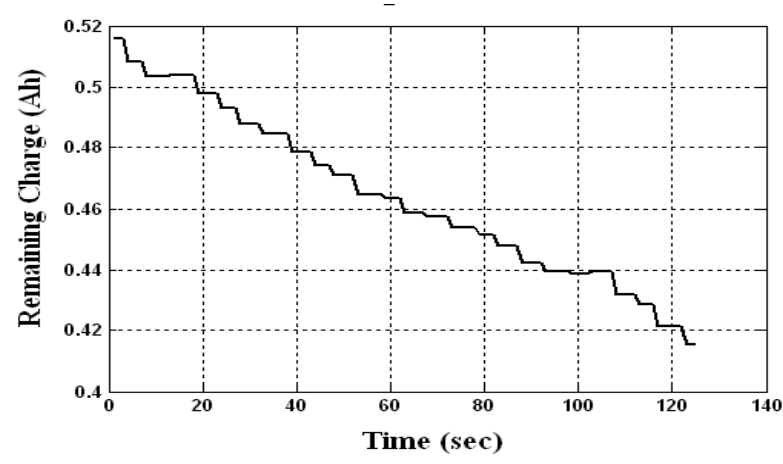

Figure 9: LMEC SOC profile in level surface at $12 \mathrm{Km} / \mathrm{hr}$

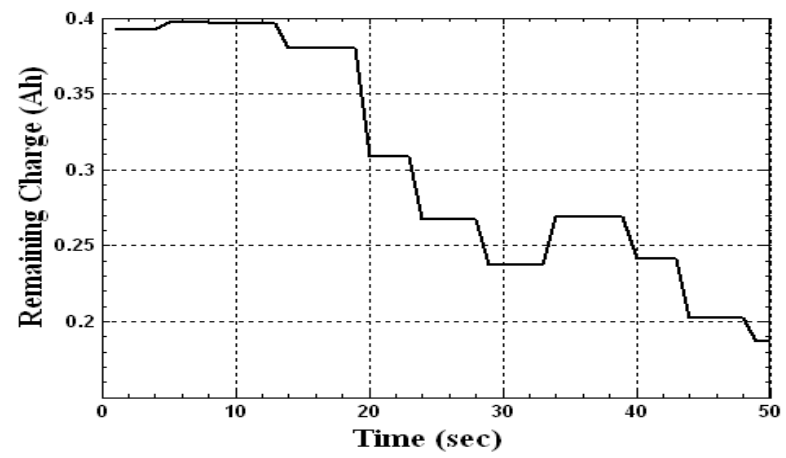

Figure 10: LMEC SOC profile in slope (25 degree) at $12 \mathrm{~km} / \mathrm{hr}$ 


\section{CONCLUSION}

The work proposes to implement an energy aware electric car using EffBMS which estimates SOC, learns the discharge profile to predict the remaining time of operation

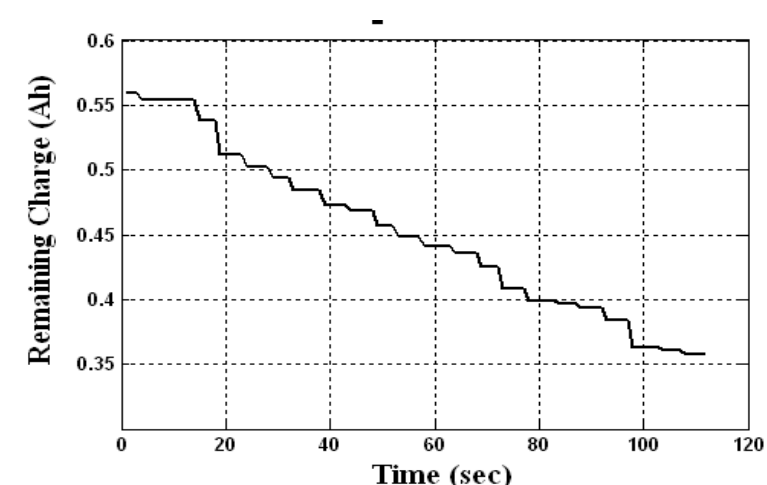

Figure 11: LMEC SOC profile in slope (25 degree) at $6 \mathrm{~km} / \mathrm{hr}$

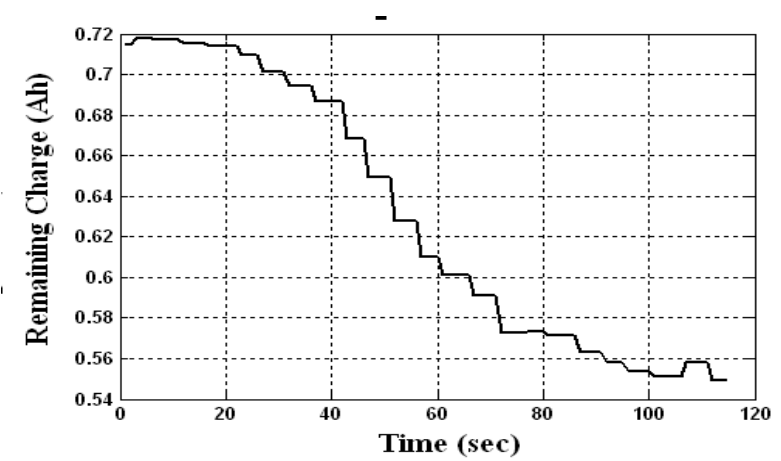

Figure 12: LMEC SOC profile using EffBMS

and optimizes the energy usage based on the learned parameters using set point controller. Based on the above analysis and discussion the following conclusions can be made:-

- The EKF based online SOC estimation gives an error of $\pm 8 \%$.

- The gradient descent based SOC learning predicts the remaining run time of the battery with tolerance $\pm 5 \%$

- The active energy management based on learned parameters is energy efficient and less costly as it does not require any additional hardware. The special case of uphill climbing is analyzed in detail and an energy saving of $12 \%$ was obtained.
The future works aims to incorporate an online battery peak power estimator to improve the dynamic load scheduling.

\section{ACKNOWLEDGEMENT}

This work was funded by CSIR-CMERI under New Millennium Indian Technology Leadership Initiative (NMITLI) Project No. 097912.

\section{REFERENCES}

[1] Dept of Heavy Industry, Govt. of India."National Mobility Mission Plan 2020", NewDelhi, 9 January,2013.

[2] K S. Ng, C.S. Moo, Y.P Chen, and Y.C Hsieh," Enhanced coulomb counting method for estimating state-of-charge and state-of-health of lithium-ion batteries." Applied energy.vol. 86, 2009.pp. 1506-1511.

[3] H. He, R. Xiong, and J. Fan."Evaluation of lithium-ion battery equivalent circuit models for state of charge estimation by an experimental approach." Energies. vol.4, 2011.pp. 582-598.

[4] W. X Shen, K.T Chau, C.C. Chan, "Neural network based residual capacity indicator for NiMH batteries in electric vehicles" IEEE Transactions of Vehicular technologies. vol.54. 2005.pp. 1705-1712..

[5] T. Hansen, C.J. Wang, "Support vector based battery state of charge estimator",Journal of power sources, vol 141.2011. pp. 351:358.

[6] W. He, N. Williard, C. Chen, M. Pecht, "State of charge estimation for electric vehicle batteries using unscented kalman filtering", Microelectronics Reliability, Available online-15 January 2013

[7] S.I Jeon, , S.T. Jo, Y.I Park, and J.M Lee. "Multi-mode driving control of a parallel hybrid electric vehicle using driving pattern recognition." Journal of dynamic systems, measurement, and controlvol. 124, 2002, pp. 141-149.

[8] C. Zhang; A.Vahidi, P.Pisu, X. Li,K. Tennant, "Role of Terrain Preview in Energy Management of Hybrid Electric Vehicles", IEEE Transactions on Vehicular Technology, vol.59,March 2010,pp.11391147.

[9] X. Huang; Y. Tan; X. He, "An Intelligent Multifeature Statistical Approach for the Discrimination of Driving Conditions of a Hybrid Electric Vehicle," Intelligent Transportation Systems, IEEE Transactions on, vol.12, June 2011, pp.453,465.

[10] A. Dardanelli. M. Tanelli, S.M. Savaresi, Mario Santucci, "Active energy management of electric vehicles with cartographic data," IEEE International Electric Vehicle Conference (IEVC), March 48 ,2012 .pp.1-6,

[11] J. Lee, O. Nam, B.H. Cho," Li-ion battery SOC estimation method based on the reduced order extended Kalman filtering", Journal of Power Sources, Volume 174, 22 November 2007, pp. 9-15..

[12] Idaho national engineering and environmental laboratory. Battery test manuel for plug in Hybrid electric vehicles. September 2010. http:/www.inl.gov/technical publications/Documents/4655291.pdf

[13] Rahmoun and H. Beichl. "Modelling of Li-ion batteries using equivalent circuit diagrams." ELEKTROTECHNICZNY (Electrical Review), 2012..ISSN 00332097.

[14] H. He, R. Xiong, H. Guo, "Online estimation of model parameters and state-of-charge of LiFePO4 batteries in electric vehicles", Applied Energy, Volume 89, Issue 1, January 2012 pp. 413-420,

[15] Andrew Ng, "CS229 Lecture notes." CS229 Lecture notes 1.1 (2000): $1-3$. 